\title{
PARÖMIE ALS TEXTORGANISATORISCHES MITTEL
}

\section{Einführung}

Ausgewählte Werbetexte aus zehn verschiedenen deutschen, österreichischen und kroatischen Zeitungen und Zeitschriften werden einer Analyse unterzogen, die Antworten geben auf die Fragen: (1) Wo im Kontext der Werbung werden Parömien eingesetzt? (2) Wie werden sie dort gebraucht?

Es konnte festgestellt werden, daß parömische Mittel eine wichtige stilistische und textorganisierende Funktion haben. Die Parömie wird zumeist variiert, d.h., weniger in der originalen Version gebraucht.

\section{Das Ziel der Untersuchung}

Aufgrund der parömiologischen Untersuchungen an verschiedenen deutschen, österreichischen und kroatischen Zeitungen in den letzten 7 Jahren hat sich herausgestellt, daß die beliebtesten Bereiche für den Gebrauch von parömischen Mitteln in der Zeitung die Politik, die Werbung und der Sport sind. ${ }^{1}$ Während in den untersuchten kroatischen Zeitungen die meisten Parömien im Kontext der Politik und des Sports vorkommen, ist die Werbung der beliebteste Kontext für die deutschen Parömien.

1 Vgl. folgende Arbeiten: Wolfgang Mieder (1979): "Sprichwörtliche Schlagzeilen in der Wochenzeitung", in: Muttersprache, XXXVIII, S.93-105; Wolfgang Mieder (1973): "Verwendungsmöglichkeiten und Funktionswerte des Sprichworts in der Wochenzeitung", in: Muttersprache, LXXXIII, S.89-119; Željka Matulina (1991): "Upotreba poslovica u sportskim novinama_s hrvatskog ili srpskog I njemačkog govornog područja", in: Novi Sad, S.216-224; Željka Matulina (1991): "Sprichwörter in Artikelüberschriften der 'Slobodna Dalmacija' und einiger vergleichbarer österreichischer Tageszeitungen", in: Znanstvena revija, 1, Maribor, S.97-108; Željka Matulina (1991): "Upotreba poslovica_u dnevnim novinama", in: (Hrsg.) Branko Tošovic "Jezik I stil u sredstvima informisanja", Sarajevo, S.48-66; Željka Matulina (1992): "Der Gebrauch von Sprichwörtern in der Zadarer Wochenzeitung 'Narodni list'", in: Proverbium 9, Vermont, USA, S.139-158; Željka Matulina (1992): "Upotreba poslovica u_naslovima austrijskog tiednika 'Wochenpresse'", in: Radovi Filozofskog fakulteta u Zadru, Heft 30, Zadar, S.69-91; Željka Matulina (1993): "Upotreba poslovica u osječkom dnevnom listu 'Glas Slavonije'", in Radovi Filozofskog fakulteta u Zadru, Heft 31, Zadar, S.155-176. 
Gerade diese Tatsache hat mich angeregt, eine ausgewählte Zahl von Werbetexten aus verschiedenen deutschen, österreichischen und kroatischen Zeitungen einer Analyse zu unterziehen, mit dem Ziel, auf zwei Fragen näher einzugehen: (1) Wo im Kontext der Werbung werden Parömien eingesetzt? und (2) Wie werden sie dort gebraucht?

\section{Der Korpus}

Um die gestellten Fragen beantworten zu können, habe ich einen Korpus von Zeitungstexten aus 10 verschiedenen Zeitungen und Zeitschriften erstellt. Der Korpus enthält mehrere Ausgaben von folgenden deutschen, österreichischen und kroatischen Zeitungen: "Školske novine" aus Zagreb, "Der Spiegel", "Burda", "Freundin" und "Die Zeit" aus Hamburg, "Westdeutsche Allgemeine Zeitung" aus Essen, "Wirtschaftswoche" und "Wochenpresse" aus Wien, "Sportske novosti" aus Zagreb und "Sport-Bild" aus Hamburg. Es handelt sich um insgesamt 11754 Seiten Zeitungen, vgl. hier die Tabelle Nr. 1.

Die Auswahl verschiedenartiger Zeitungen ist absichtlich gemacht worden. Werbung ist in verschiedenen Zeitungen nicht gleich vertreten. Es gibt Zeitungen mit mehr Werbung darin, es gibt Zeitungen mit weniger Werbung. Die Anwesenheit von Werbetexten in einer Zeitung ist vom Inhalt der Zeitung und von ihren Adressaten abhängig. Es spielen aber auch die ökonomischen und marktwirtschaftlichen Interessen und die finanziellen Möglichkeiten des betreffenden Landes eine Rolle. Für die kroatischen Zeitungen bzw. Zeitschriften gilt im Allgemeinen, daß in ihnen viel weniger von der Reklame Gebrauch gemacht wird als in den entsprechenden österreichischen oder deutschen Zeitungen/Zeitschriften. So sind z.B. in den kroatischen Zeitungen "Školske novine" und "Sportske novosti" keine Werbetexte zu finden im Unterschied etwa zur deutschen speziellen Zeitung "Sport-Bild". Vgl. auch die Tabelle Nr. 2. Aus der Analyse der zur Verfügung stehenden Texte geht hervor, daß die beiden österreichischen Zeitschriften "Wochenpresse" und "Wirtschaftswoche" von der Werbung und dementsprechend von der Parömie am meisten Gebrauch machen. Ganz stark in parömiologischer Hinsicht ist daneben auch die "Westdeutsche Allgemeine Zeitung", wie das der tabellarischen Übersicht Nr. 1 zu entnehmen ist.

\section{Wo und wie werden Parömien im Werbetext verwendet?}

Parömien trifft man vor allem in Titeln der Zeitungsartikel bzw. in Überschriften der Werbungsannoncen. ${ }^{2}$ Von allen Sprichwörtern in Titeln/Überschriften im Kontext

2 In eine detailliertere Abgrenzung zwischen den beiden Begriffen "Titel" und "Überschrift" ist in dieser Arbeit nicht eingegangen worden. 
der Werbung erscheinen $73 \%$ Sprichwörter und 27\% Sprichwörter innerhalb der Zeitungsartikel. Hier ein paar Beispiele:

\section{Lachende Dritte}

(WW, 1994; Quelle: Wer zuletzt lacht, lacht am besten. Wenn zwei sich streiten, freut sich der Dritte.)

Unkraut verdirbt nicht

(WP, 1989; Quelle: Unkraut verdirbt nicht. Es handelt sich um einen Werbetext, in dem über das Konzert der Musikgruppe "Brennesseln" die Rede ist.)

Der Klügere liest nach

(SPIE, 1994; Quelle: Der Klügere gibt nach. Es wird für eine Zeitung geworben.)

Wohnen und leben lassen

(Zeit, 1994; Quelle: Leben und leben lassen. Werbung für Möbel.)

Warme Füße! Alles gut!

(FREU, 1993; Quelle: Ende gut, alles gut! Werbung für Damenstrümpfe.)

Verändern ist Silber. Verbessern ist Golf.

(SB, 1989; Quelle: Reden ist Silber, Schweigen ist Gold. Werbung für die Automobilindustrie.)

Im Titel/in der Überschrift wird die ursprüngliche Form der Parömie gerne geändert. Sehr häufig kommen Veränderungen im lexikalischen Bereich vor. So wird z.B. ein Wort der Parömie oft durch ein außerhalb der Parömie genommenes Element ersetzt. Meistens steht gerade in diesem neuen Element der Name des Produkts, wofür mit dem Werbetext bzw. mit der Überschrift geworben wird. Von insgesamt 77 Parömien in den Titeln der Werbetexte aus unserem Korpus werden 39 Parömien auf diese Art und Weise umformuliert - das ist der sog. III Strukturtyp. Vgl. hier die Tabelle Nr. 4.

Es scheint wichtig, zuerst eine Übersicht über die bis jetzt festgestellten Typen der formalen Variierung von Parömien zu geben. ${ }^{3}$

I Typ

Die originale Form der Parömie wird beibehalten.

II Typ

Man übernimmt das syntaktische Modell der ursprünglichen Parömie und die Lexik ist vollkommen geändert.

3 Eine Unterscheidung bzw. Klassifikation von verschieden strukturierten Werbetexten ist hier nicht gemacht worden. Es ist aber offensichtlich, daß es Werbetexte gibt, die aus nur einem Satz bestehen, z.B. aus einer Überschrift und einem Photo, oder aus zwei Überschriften und einem Photo, usw. 


\section{Typ}

Man übernimmt das syntaktische Modell der ursprünglichen Parömie, aber die Lexik ist nur teilweise geändert; nur ein Bestandteil der Parömie wird durch ein außerhalb der Parömie genommenes Element ersetzt.

IV Typ

Partielle oder völlige Umstellung der innerhalb der Parömie schon vorhandenen Bestandteile.

\section{Typ}

Das syntaktische Modell ist geändert, aber die Lexik ist völlig erhaiten.

VI Typ

Das syntaktische Modell und die Lexik sind beide geändert; wenigstens ein Element entweder vom syntaktischen Modell der ursprünglichen Parömie oder von ihrer Lexik muß erhalten bleiben, um die ursprüngliche Parömie assoziieren zu können.

VII Typ

Kombination zweier Parömien oder einer Parömie und einer anderen verwandten Art (z.B. einer Redewendung).

VIII Typ

Ein Teil der ursprünglichen Parömie wird eliminiert.

\section{Typ}

Verschiedene Erweiterungen der Parömie werden vorgenommen, z.B. Einfügung eines neuen Elements oder komplexerer Einheiten vor oder nach der Parömie (prä- und postpositionierte Erweiterungen der Parömie).

Und wo sind Parömien im Werbetext selbst loziert? Wie sehen sie dort aus?

Um die zur Verfügung stehenden Werbetexte durchsichtig machen zu können und sie dementsprechend analysieren und beschreiben zu können, habe ich jeden Text in drei quantitativ gleiche Abschnitte eingeteilt - in drei Drittel des jeweiligen Textes: in das erste Drittel des Textes, worin die Einführung in den Gesamttext steckt, in das zweite Drittel oder den mittleren Teil des Textes und in das dritte Drittel des Textes, der den Schluß des gesamten Werbetextes enthält.

\section{Das erste Drittel des Textes}

Aus der Analyse des ersten Drittels des Textes geht hervor, daß die Parömie oft schon im ersten Satz erscheint. Von insgesamt 29 Sätzen mit Parömien, stehen 10 Sätze gerade am Anfang des gesamten Textes. Das bedeutet statistisch mehr als ein Drittel aller Fälle, wie es in der Tabelle Nr. 3 steht.

Auch hier kann man feststellen, daß die Parömie gerne variiert wird. Nur in 4 von insgesamt 10 Fällen erscheint hier die Parömie in ihrer originalen Form (I Typ). Der III Typ ist in diesem Teil des Zeitungsartikels meistens vertreten, Das heißt, daß hier das Sprichwort am liebsten durch eine Substitution im lexikalischen Bereich geändert wird. Neben dem III Typ erscheint hier oft auch der IX Typ und dessen Kombination mit an- 
deren Typen, was bedeutet, daß das Sprichwort gerne durch einen vor- oder nachgestellten Satz erweitert wird (vgl. Tabelle Nr. 6). Hier einige Beispiele:

Sind Sie schon einmal vom Himmel gefallen? Nein? Dann sind Sie auf dem besten Weg, ein Meister zu werden.

(WP, 1989; Quelle: Es ist noch kein Meister vom Himmel gefallen.)

Wo gehobelt wird, das weiß jeder, fliegen Späne.

(WP, 1989; Quelle: Wo gehobelt wird, da fallen Späne.)

So viele Sprachen du sprichst, soll der alte Geheimrat Goethe einmal gesagt haben, so oft lebst du.

(WP, 1988; Quelle: Wie viele Sprachen du sprichst, so vielen Menschen du giltst; Der erste Satz der Werbung für "Superlearning Sprachkurse".)

Auch hier gilt im allgemeinen, daß die zu erweiternde Parömie schon eine Variation der Quelle ist.

\section{Das zweite Drittel des Textes}

Im mittleren Teil des Werbetextes scheinen die parömischen Mittel nicht besonders gerne gebraucht zu werden. Im untersuchten Korpus erscheinen nur 2 Parömien in diesem Teil des Textes und zwar als erster Satz (vgl. die Tabelle Nr. 7). Der VI Typ der strukturellen Umformulierung ist der einzige, der in diesem Teil des Textes erscheint. Das bedeutet, daß die Parömie so stark variiert wird, daß von ihr nur ein Element der lexikalischen bzw. syntaktischen Mittel noch geblieben ist, um die ursprüngliche Form der Parömie zu assoziieren.

Zum Glück bieten Thnen alle unsere Drucker so viele dieser Vorteile, daß die Wahl ganz sicher nicht zur Qual wird.

(WW, 1993; Quelle: Wer die Wahl hat, hat die Qual.)

\section{Das dritte Drittel des Textes}

Im letzten Drittel des Werbetextes erscheinen 13 Parömien und davon sind $10 \mathrm{im}$ letzten Satz loziert, vgl. die Tabelle Nr. 3. Das bestätigt die These, daß die Parömie eine schlußfolgernde textabschließende, gleichzeitig auch eine resümierende, konklusive Funktion im Text hat.

Auch hier erscheint die Parömie nicht in ihrer originalen Form. In diesem Teil des Zeitungstextes überwiegen der IX Typ und die Kombination des IX mit dem III Typ. Das bedeutet, daß das Sprichwort durch einen Satz erweitert wird. Diese Erweiterung erfolgt von vorne (prä-positionierter Satz), von hinten (post-positionierter Satz) aber auch eingeschobene Sätze sind häufig in Rahmen dieses Typs.

Hier einige Beispiele für die Erweiterung durch einen prä-positionierten Satz:

Für diejenigen, die der neuen Lernmethode immer noch skeptisch gegenüberstehen...haben wir schließlich noch ein Sprichwort parat: Probieren geht über Studieren. 
(WP, 1989; Quelle: Probieren geht über Studieren; der allerletzte Satz des Textes, womit für die neue Fremdsprachen-Lernmethode geworben wird.)

Bei alldem sollten Sie jedoch nicht aufs Aufhören vergessen und sich hie und da an die alte Managerweisheit erinnern, wonach Zeit Geld ist.

(WP, 1988; Quelle: Zeit ist Geld.)

Die Erweiterung kann aber auch hinter der Parömie vorkommen;

Gutes Rad ist teuer, unser Rat kostenlos: Eine Leserclubmitgliedschaft zahlt sich aus.

(WP, 1988; Quelle: Guter Rat ist teuer; Werbung für eine Walkie-Talkie-Anlage fürs Fahrrad "Bikeman".)

\section{Schlußwort}

(a) Zusammenfassend kann man sagen, daß die parömischen Mittel innerhalb eines Werbetextes nicht zufällig erscheinen. Sie haben in einem solchen Text eine sehr wichtige stilistische und textorganisatorische Funktion. Über ihre stilistischen Werte zeugen Variationen im strukturell-syntaktischen und lexikalischen Bereich, über ihren textorganisatorischen Charakter ihre Lozierung im Text.

In Titeln der Werbetexte erscheinen Parömien 77 mal, was $28 \%$ von allen Titeln im ganzen untersuchten Korpus ausmacht. In Zeitungsartikeln kommt die Parömie $10 \mathrm{mal}$ als erster Satz des Textes vor und das bedeutet 35\% von allen Parömien in Werbetexten. Auch im letzten Satz des Textes kommen 10 Parömien vor, und das sind wieder 35\% von allen Parömien in Werbetexten.

Die Lozierung der Parömie im Text der Werbung kann durch die folgende Graphik veranschaulicht werden:

PARÖMIE INNERHALB DES ZEITUNGSARTIKELS

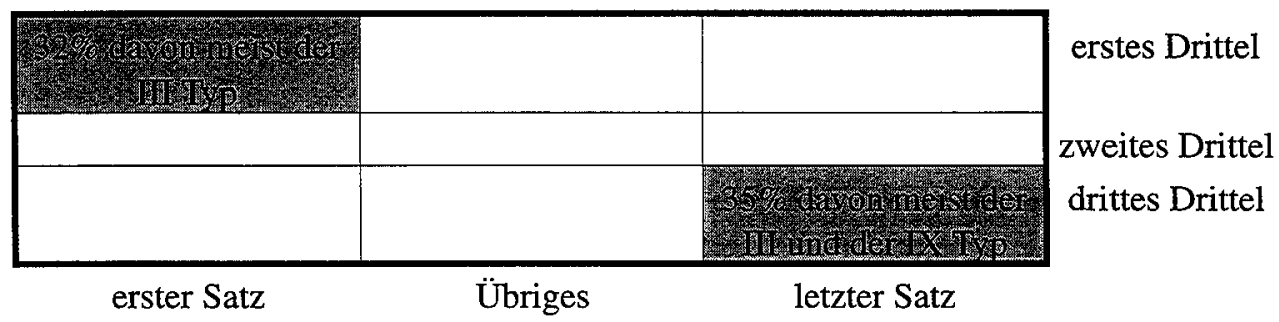

(b) Was die Form der Parömie im Werbetext anbetrifft, wird sie weniger in der originalen Version gebraucht und mehr als Variation der Quelle. Am häufigsten scheinen in (untersuchten) Texten die strukturellen Umformulierungen des III und des IX Typs bzw. deren Kombination zu sein. Das heißt, daß meist das syntaktische Modell der 
Quelle übernommen wird und die Lexik teilweise verändert wird. Oder es wird die auf diese Art und Weise umformulierte Parömie noch weiter erweitert. Das geschieht, wenn neue Sätze oder Wortgruppen vor oder hinter die Parömie zugefügt oder zwischen die Teile der Parömie eingeschoben werden.

(c) Es können zuletzt die im Korpus der Werbetexte häufigst gebrauchten Parömien genannt werden (hier in ihrer originalen Form):

1 Liebe geht durch den Magen. (11 mal)

2 Aus den Augen, aus dem Sinn. (6 mal)

3 Kein Meister ist vom Himmel gefallen. (4 mal)

4 Veni, vidi, vici. (4 mal)

5 Wer die Wahl hat, hat die Qual. (3 mal)

6 Sicher ist sicher. (3 mal)

7 Ende gut, alles gut. ( $3 \mathrm{mal})$

8 Guter Rat ist teuer. ( 3 mal)

9 Dabeisein ist alles. $(3 \mathrm{mal})$

10 Frïh übt sich, wer ein Meister werden will. (3 mal)

\section{ABKÜRZUNGEN}

$\begin{array}{llll}\text { SN } & \text { "Školske novine" } & \text { WAZ } & \text { "Westdeutsche Allgemeine Zeitung" } \\ \text { SPIE } & \text { "Der Spiegel" } & \text { WW } & \text { "Wirtschaftswoche" } \\ \text { BUR } & \text { "Burda" } & \text { WP } & \text { "Wochenpresse" } \\ \text { FREU } & \text { "Freundin" } & \text { SN } & \text { "Sportske novine" } \\ \text { ZEIT } & \text { "Die Zeit" } & \text { SB } & \text { "Sport-Bild" }\end{array}$

\section{LITERATUR}

Beyer, Horst und Annelies (1986) : Sprichwortlexikon, Verlag C.H.Beck, München Röhrich, Lutz / Mieder, Wolfgang (1977): Sprichwort, J.B.Metzlersche Verlagsbuchhandlung, Stuttgart Simrock, Karl (1988): Die deutschen Sprichwörter, Reclam, Stuttgart Skarpa, Vicko (1909): Hrvatske narodne poslovice, Sibenik 
Tabelle Nr. 1: ALLGEMEINE ANGABEN ÜBER DEN KORPUS

\begin{tabular}{|c|c|c|c|c|c|c|c|c|c|c|c|}
\hline Name der Zeitung & $\frac{\text { Skolske }}{\text { novine }}$ & $\begin{array}{c}\text { Der } \\
\text { Spiegel } \\
\text { (SPIE) } \\
\text { Hamburg }\end{array}$ & $\begin{array}{c}\text { Burda } \\
\text { (BUR) } \\
\text { Hamburg }\end{array}$ & $\begin{array}{l}\text { Ereundin } \\
\text { (FREU) } \\
\text { Hamburg }\end{array}$ & $\begin{array}{c}\text { Die Zeit } \\
\text { (ZEIT) } \\
\text { Hamburg }\end{array}$ & $\begin{array}{l}\frac{\text { Westdeutsch }}{\mathrm{e}} \\
\text { Allgemeine } \\
\text { Zeitung } \\
\text { (WAZ) } \\
\text { Essen }\end{array}$ & $\begin{array}{l}\frac{\text { Wirtschafts- }}{\text { woche }} \\
\text { (WW) Wien }\end{array}$ & $\begin{array}{l}\frac{\text { Wirtschafts- }}{\text { presse }} \\
\text { (WP) Wien }\end{array}$ & $\begin{array}{c}\text { Sportske } \\
\text { novosti } \\
\text { (SN) } \\
\text { Zagreb }\end{array}$ & $\begin{array}{c}\text { Sport-Bild } \\
\text { (SB) } \\
\text { Hamburg }\end{array}$ & insgesamt \\
\hline Art der Zeitung & $\begin{array}{l}\text { Wochen- } \\
\text { zeitung }\end{array}$ & $\begin{array}{l}\text { Wochen- } \\
\text { zeitung }\end{array}$ & $\begin{array}{l}\text { Monats- } \\
\text { zeitschrift }\end{array}$ & $\begin{array}{l}\text { erscheint } \\
\text { alle zwei } \\
\text { Wochen }\end{array}$ & $\begin{array}{l}\text { Wochen- } \\
\text { zeitung }\end{array}$ & Tageszeitung & $\begin{array}{l}\text { Wochen- } \\
\text { zeitschrift }\end{array}$ & $\begin{array}{l}\text { Wochen- } \\
\text { zeitschrift }\end{array}$ & $\begin{array}{l}\text { Tages- } \\
\text { zeitung }\end{array}$ & $\begin{array}{l}\text { Wochen- } \\
\text { illustrierte }\end{array}$ & \\
\hline Inhalt der Zeitung & $\begin{array}{l}\text { Pädagogik, } \\
\text { Unterricht }\end{array}$ & $\begin{array}{l}\text { Politik, } \\
\text { Wirtschaft, } \\
\text { Kultur }\end{array}$ & Frauenmode & Frauenmode & $\begin{array}{l}\text { Politik, } \\
\text { Wirtschaft, } \\
\text { Kultur }\end{array}$ & $\begin{array}{l}\text { Politik, } \\
\text { Wirtschaft, } \\
\text { Kultur, } \\
\text { Sport }\end{array}$ & Wirtschaft & $\begin{array}{l}\text { Politik, } \\
\text { Wirtschaft, } \\
\text { Kultur }\end{array}$ & Sport & Sport & \\
\hline $\begin{array}{l}\text { Zahl der analysierten Ausga- } \\
\text { ben }\end{array}$ & 14 & 11 & 14 & 2 & 8 & 25 & 15 & 35 & 19 & 13 & \\
\hline Jahrgang & 1993,1994 & 1994 & 1993,1994 & 1994 & 1994 & 1991 & 1993,1994 & $1987-1990$ & 1989 & 1989 & \\
\hline Zahl der Seiten einer Ausgabe & 16 & ca. 250 & ca. 180 & ca. 180 & $\begin{array}{c}\text { ca. } 140+ \\
\text { ca. } 50 \\
\text { (Beilage) } \\
\end{array}$ & ca. 90 & ca. 90 & ca. 80 & 16 & ca. 50 & $\begin{array}{l}\text { ca. } 11574 \\
\text { Seiten }\end{array}$ \\
\hline $\begin{array}{l}\text { Gesamtzahl der festgestellten } \\
\text { Sprichwörter }\end{array}$ & 9 & 14 & 3 & 4 & 13 & 33 & 39 & 105 & 36 & 14 & 270 \\
\hline $\begin{array}{l}\text { Zahl der Sprichwörter im } \\
\text { Kontext der Werbung }\end{array}$ & 0 & $8(=57 \%)$ & 1 & 2 & 4 & $19(=58 \%)$ & $21 \Leftrightarrow 54 \%)$ & $44(=42 \%)$ & 0 & $7(=50 \%)$ & 106 \\
\hline
\end{tabular}


Tabelle Nr. 2: SPRICHWÖRTER IM KONTEXT DER WERBUNG

\begin{tabular}{|c|c|c|c|c|c|c|c|c|c|c|c|}
\hline Zeitung & ŠN & SPIE & BUR & FREU & ZEIT & WAZ & WW & WP & SN & SB & insgesamt \\
\hline Sprichwörter in Titeln/ Überschriften & 6 & 10 & 3 & 4 & 8 & 18 & 36 & 66 & 8 & 12 & 171 \\
\hline Davon nur im Kontext der Werbung & 0 & 8 & 1 & 2 & 3 & 16 & 19 & 22 & 0 & 6 & 77 \\
\hline Prozent in Titeln/ Überschriften & $0 \%$ & $80 \%$ & $33 \%$ & $50 \%$ & $37 \%$ & $89 \%$ & $51 \%$ & $33 \%$ & $0 \%$ & $46 \%$ & $45 \%$ \\
\hline Davon nur im Kontext der Werbung & 0 & 0 & 0 & 0 & 1 & 3 & 2 & 22 & 0 & 1 & 29 \\
\hline Prozent innerhalb der Artikel & $0 \%$ & $0 \%$ & $0 \%$ & $0 \%$ & $20 \%$ & $20 \%$ & $67 \%$ & $56 \%$ & $0 \%$ & $50 \%$ & $29 \%$ \\
\hline Sprichwörter insgesamt & 9 & 14 & 3 & 4 & 13 & 33 & 39 & 105 & 36 & 14 & 270 \\
\hline
\end{tabular}

Tabelle Nr. 3: LOZIERUNG DES SPRICHWORTS IM KONTEXT DER WERBUNG

\begin{tabular}{|c|c|c|c|c|c|c|c|c|c|c|c|c|c|}
\hline \multicolumn{2}{|l|}{ Zeitung } & ŠN & SPIE & BUR & FREU & ZEIT & WAZ & Ww & WP & SN & SB & insgesamt & Prozent \\
\hline \multicolumn{2}{|c|}{ Sprichwort im Titel/ in der Überschrift } & 0 & 8 & 1 & 2 & 3 & 16 & 19 & 22 & 0 & 6 & $\begin{array}{c}77 \\
(=28 \%)\end{array}$ & $28 \%$ \\
\hline \multicolumn{14}{|c|}{ Sprichwort innerhalb des Zeitungsartikels } \\
\hline \multirow{2}{*}{$\begin{array}{l}\text { ERSTES DRITTEL DES AR- } \\
\text { TIKELS }\end{array}$} & letzter Satz & & & & & & & & & & & & \multirow{2}{*}{$\begin{array}{l}14 \\
\text { Sprichwörter oder } \\
50 \%\end{array}$} \\
\hline & Übriges & & & & & & & & 3 & & 1 & 4 & \\
\hline $\begin{array}{l}\text { ZWEITES DRITTEL DES } \\
\text { ARTIKELS }\end{array}$ & Übriges & & & & & & & & & & & & $\begin{array}{l}2 \\
\text { Sprichwörter }\end{array}$ \\
\hline \multirow{3}{*}{$\begin{array}{l}\text { DRITTES DRITTEL DES } \\
\text { ARTIKELS }\end{array}$} & erster Satz & & & & & & & & & & & & \multirow{3}{*}{$\begin{array}{l}13 \\
\text { Sprichwörter oder } \\
45 \%\end{array}$} \\
\hline & letzter Satz & & & & & & & & 10 & & & & \\
\hline & Übriges & & & & & & & & 3 & & & & \\
\hline $\begin{array}{l}\text { insgesamt innerhalb der Ar- } \\
\text { tikel }\end{array}$ & & 0 & 0 & 0 & 0 & 1 & 3 & 2 & 22 & 0 & 1 & $\begin{array}{c}29 \\
(=10 \%)\end{array}$ & \\
\hline
\end{tabular}


Tabelle Nr. 4: FORM DES SPRICHWORTS IN TITELN/ ÜBERSCHRIFTEN

\begin{tabular}{|c|c|c|c|c|c|c|c|c|c|c|c|}
\hline Zeitung Strukturtyp & SN & SPIE & BUR & FREU & ZEIT & WAZ & Ww & WP & SN & SB & insgesamt \\
\hline I & 5 & 1 & & 1 & 7 & 2 & 2 & & & 18 & \\
\hline \multicolumn{12}{|l|}{ II } \\
\hline III & & 3 & & & 2 & 4 & 12 & 13 & & 5 & $39(=50 \%)$ \\
\hline \multicolumn{12}{|l|}{ IV } \\
\hline \multicolumn{12}{|l|}{$\mathrm{V}$} \\
\hline VI & & & & 2 & & 1 & 5 & 1 & & 1 & 10 \\
\hline \multicolumn{12}{|l|}{ VII } \\
\hline VIII & & & & & & 2 & & 2 & & & 4 \\
\hline IX & & & & & & 2 & & 1 & & & 3 \\
\hline $\begin{array}{l}\text { Kombinationen mehrerer } \\
\text { Strukturtypen }\end{array}$ & & & & & & & & 3 & & & 3 \\
\hline insgesamt & 0 & 8 & 1 & 2 & 3 & 16 & 19 & 22 & 0 & 6 & 77 \\
\hline
\end{tabular}

Tabelle Nr. 5: FORM DES SPRICHWORTS INNERHALB DER ZEITUNGSARTIKEL IM KONTEXT DER WERBUNG

\begin{tabular}{|c|c|c|c|c|c|c|c|c|c|c|c|}
\hline Zeitung Strukturtyp & SN & SPIE & BUR & FREU & ZEIT & WAZ & Ww & WP & SN & SB & insgesamt \\
\hline I & & & & & 1 & 2 & & 3 & & & 6 \\
\hline \multicolumn{12}{|l|}{ II } \\
\hline III & & & & & & & & 5 & & & 5 \\
\hline \multicolumn{12}{|l|}{ IV } \\
\hline \multicolumn{12}{|l|}{$\mathrm{V}$} \\
\hline VI & & & & & & & 2 & & & & 2 \\
\hline \multicolumn{12}{|l|}{ VII } \\
\hline VIIII & & & & & & & & 1 & & & 1 \\
\hline IX & & & & & & 1 & & 5 & & 1 & 7 \\
\hline $\begin{array}{l}\text { Kombinationen mehrerer } \\
\text { Strukturtypen }\end{array}$ & & & & & & & & 8 & & & 8 \\
\hline insgesamt & 0 & 0 & 0 & 0 & 1 & 3 & 2 & 22 & 0 & 1 & 29 \\
\hline
\end{tabular}


Tabelle Nr. 6: FORM DES SPRICHWORTS IM ERSTEN DRITTEL DES ZEITUNGSARTIKELS

\begin{tabular}{|l|c|c|c|c|c|}
\hline Zeitung & ZEIT & WAZ & WP & SB & insgesamt \\
\hline erster Satz & I & IX, I, I & III + IX, III & III + IX, III & VI + IX, III \\
\hline insgesamt & & & & & \\
\hline letzter Satz & 1 & 3 & 6 & & 0 \\
\hline insgesamt & & & & & \\
\hline Übriges & & & III + IX, IX, I & IX & 1 \\
\hline insgesamt & & & 3 & 1 & 14 \\
\hline
\end{tabular}

Tabelle Nr. 7: FORM DES SPRICHWORTS IM ZWEITEN DRITTEL DES ZEITUNGSARTIKELS

\begin{tabular}{|l|c|c|}
\hline Zeitung & WW & insgesamt \\
\hline erster Satz & VI, VI & 2 \\
\hline insgesamt & 2 & \\
\hline letzter Satz & & \\
\hline insgesamt & & \\
\hline Übriges & & \\
\hline insgesamt & & \\
\hline
\end{tabular}

Tabelle Nr. 8: FORM DES SPRICHWORTS IM DRITTEN DRITTEL DES ZEITUNGSARTIKELS

\begin{tabular}{|l|c|c|}
\hline Zeitung & WP & insgesamt \\
\hline erster Satz & & \\
\hline insgesamt & & \\
\hline letzter Satz & I, VIII & III + IX, III + IX \\
\hline insgesamt & 10 & \\
\hline Übriges & VI + IX, IX, III & 3 \\
\hline insgesamt & 3 & 13 \\
\hline
\end{tabular}


Analiza izbranih besedil iz desetih različnih nemških, avstrijskih in hrvaških časopisov in revij daje odgovor na vprašanji: (1) Kje se v kontekstu reklame pojavljajo pregovori? (2) Kako se uporabljajo pregovori v reklamah?

Ugotoviti je bilo mogoče, da imajo pregovori pomembno stilistično funkcijo kot tudi funkcijo oblikovanja besedila. Pregovor se večinoma uporablja spremenjeno, t.p. manj $v$ originalni verziji. 